\title{
Epipericardial fat necrosis - a rare cause of pleuritic chest pain: case report and review of the literature
}

\author{
Thomas Runge ${ }^{1}, M$. Andrew Greganti²
}

1Student at the University of North Carolina School of Medicine and the Gillings School of Global Public Health, Chapel Hill, NC, USA

2Department of Medicine at the University of North Carolina School of Medicine, in Chapel Hill, NC, USA

Submitted: 11 May 2010

Accepted: 12 July 2010

Corresponding author: Thomas Runge MD, MPH

201 Chimeneas Place Chapel Hill, NC 27517

Phone: (919): 824-6002

E-mail:

thomas.m.runge@gmail.com

Arch Med Sci 2011; 7, 2: 337-341

DOI: 10.5114/aoms.2011.22088

Copyright @ 2011 Termedia \& Banach

\begin{abstract}
Epipericardial fat necrosis (EPFN) is an uncommon cause of chest pain. Typically manifesting as severe acute chest pain, EPFN can be mistaken for a serious disorder, such as pulmonary embolism or myocardial infarction. We report a case of EPFN, diagnosed tentatively based on clinical and radiographic findings, with documented resolution of the lesion on chest CT. According to our literature search, this is only the second case in which chest CT alone was used to both diagnose the lesion and track its resolution. It is the first documented case diagnosed and followed as such in North America.
\end{abstract}

Key words: fat necrosis, pleuritic, chest pain.

\section{Introduction}

Epipericardial fat necrosis (EPFN) is an uncommon cause of chest pain. Typically manifesting as severe acute chest pain, EPFN can be mistaken for a serious disorder, such as pulmonary embolism or myocardial infarction. We report a case of epipericardial fat necrosis, diagnosed tentatively based on clinical and radiographic findings, with documented resolution of the lesion on chest CT. According to our literature search, this is only the second case in which chest CT alone was used to both diagnose the lesion and track its resolution. It is the first documented case diagnosed and followed as such in North America.

\section{Case report}

A 70-year-old man with a history of borderline hypertension and mild obesity was admitted with a 2-day history of left-sided pleuritic chest pain, left shoulder pain, and mild dyspnea. On the night of admission he awakened with severe pleuritic pain in the left mid-axillary area. The pain was not positional and did not radiate, and was not reproducible by palpation. He had mild dyspnea which he attributed to splinting to control the severity of his pain. There was no associated diaphoresis, palpitation, leg tenderness/pain, syncope, or near syncope. He did not experience any substernal burning, nausea, emesis, anorexia, or other gastrointestinal symptoms. He had been on a long airplane flight two weeks prior to his presentation. His past medical history was notable for borderline 

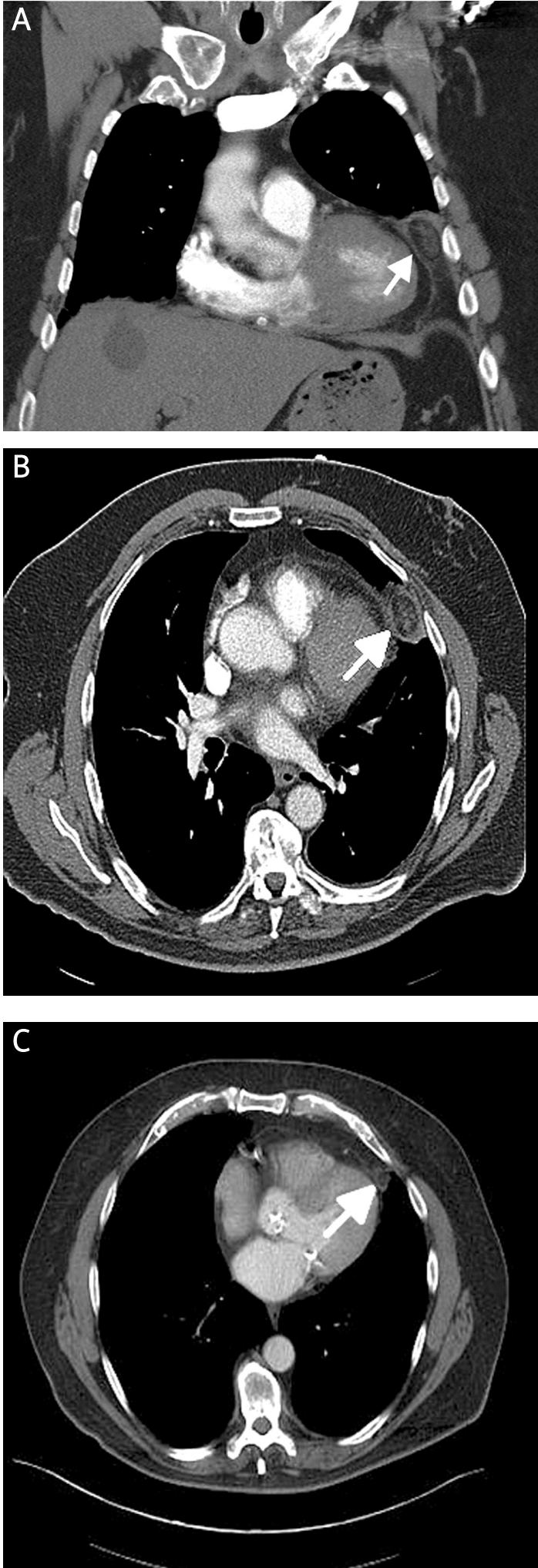

Figure 1. Chest CT: A) Coronal section shows low attenuation of the pericardial fat (arrow). An area of pathologic fat necrosis and inflammation is seen lateral to the pericardial fat, corresponding to the shadow seen on admission CXR. An axial section (B), shows the necrotic area (arrow). Six months later (C), the lesion measured $1.4 \mathrm{~cm}$ hypertension, gout, remote peptic ulcer disease, and mild obesity. He was a nonsmoker and did not consume alcohol or use illicit drugs. He reported being active with a daily exercise program including daily treadmill exercise, with no noted changes in exercise tolerance or capacity. On evaluation, his blood pressure was $156 / 90 \mathrm{mmHg}$ with a pulse of 81 and regular. He was afebrile, there were no palpable nodes, and cardiac rate and rhythm were normal, as were chest and lung exams. ECG showed normal sinus rhythm, Q waves in lead III, and nonspecific ST and T-wave abnormalities, with no evidence of acute ischemia. Cardiac enzymes on admission were CK $87 \mathrm{U} / \mathrm{L}$ (nl: 70-185), CK-MB $3.7 \mathrm{ng} / \mathrm{ml}$ ( $\mathrm{nl}$ : 0.0-6.0), and troponin $\mathrm{T}$ was undetectable ( $\mathrm{nl}:<0.029 \mathrm{ng} / \mathrm{ml})$. Eight $\mathrm{h}$ later, $\mathrm{CK}$ and CK-MB had decreased to $83 \mathrm{U} / \mathrm{l}$, and $3.1 \mathrm{ng} / \mathrm{ml}$, respectively, and troponin $\mathrm{T}$ remained undetectable. A final troponin $\mathrm{T}$ level was measured and was undetectable. Given unequivocal cardiac enzyme levels and ECG findings, the clinical suspicion for myocardial ischemia was very low. Accordingly, no additional diagnostic tests for $\mathrm{MI}$ were ordered. Chest radiograph revealed a soft tissue density adjacent to the left heart border. Chest CT identified a 6.4 by $2.5 \mathrm{~cm}$ low-density softtissue shadow in the left lung base. A second, smaller paracardiac density $(2.9 \times 2 \mathrm{~cm}$ in diameter $)$ was also noted. The presumptive diagnosis of epipericardial fat pad necrosis was made based on these findings (Figures $1 A-B$ ). The patient was admitted, observed overnight, and discharged the following morning, his pain having resolved.

Three months following discharge, the patient experienced recurrent left pleuritic chest pain. An ECG performed at this time was unchanged compared with the prior ECG, with no evidence of ischemia. Initial cardiac enzymes: CK $70 \mathrm{U} / \mathrm{l}, \mathrm{CK}-\mathrm{MB}$ $3.2 \mathrm{ng} / \mathrm{ml}$, troponin T undetectable. At $8 \mathrm{~h}$, enzymes were: CK $44 \mathrm{U} / \mathrm{l}, \mathrm{CK}-\mathrm{MB} 2.6 \mathrm{ng} / \mathrm{ml}$, troponin T undetectable. Final cardiac enzymes: CK $34 \mathrm{U} /$, CK-MB $2.6 \mathrm{ng} / \mathrm{ml}$, troponin T undetectable. Imaging studies showed that the area of increased attenuation in the paracardiac fat had decreased in size, to $2.2 \mathrm{~cm}$ by $1.4 \mathrm{~cm}$. The left-basilar density had resolved. Six months later, the paracardiac lesion had decreased in size to $1.4 \mathrm{~cm}$ in diameter (Figure 1C). The patient was monitored with serial CT imaging studies for 12 months after his acute episode, and his symptoms did not recur.

\section{Discussion}

Epipericardial fat necrosis is an uncommon cause of chest pain. Typically manifesting as severe acute chest pain, EPFN can be mistaken for a serious disorder, such as pulmonary embolism or myocardial infarction. Diagnosis has generally 
Table I. Previous cases of epipericardial fat necrosis

\begin{tabular}{|c|c|c|c|c|c|c|c|c|}
\hline Case & First author & Year* & $\begin{array}{c}\text { Age } \\
\text { [years] }\end{array}$ & Sex & $\begin{array}{c}\mathrm{BMI} \\
{\left[\mathrm{kg} / \mathrm{m}^{2}\right]}\end{array}$ & Complaint & Size $[\mathrm{cm}]$ & Treatment \\
\hline 1 & Jackson & 1957 & 52 & M & 29.9 & $\begin{array}{l}\text { Right-sided } \\
\text { chest pain }\end{array}$ & $5 \times 2.5 \times 2$ & $\begin{array}{l}\text { Exploratory } \\
\text { thoracotomy }\end{array}$ \\
\hline 2 & Jackson & 1957 & 63 & $M$ & 31.6 & $\begin{array}{l}\text { Left-sided } \\
\text { chest pain }\end{array}$ & $8 \times 4.5 \times 2.5$ & $\begin{array}{l}\text { Exploratory } \\
\text { thoracotomy }\end{array}$ \\
\hline 3 & Jackson & 1957 & 47 & $\mathrm{~F}$ & 39.4 & $\begin{array}{l}\text { Left-sided } \\
\text { chest pain }\end{array}$ & $7.4 \times 6 \times 2.5$ & $\begin{array}{l}\text { Exploratory } \\
\text { thoracotomy }\end{array}$ \\
\hline 4 & Kasserman & 1958 & 23 & M & 21.9 & $\begin{array}{l}\text { Left-sided } \\
\text { chest pain }\end{array}$ & $7 \times 4.5 \times 2$ & $\begin{array}{l}\text { Exploratory } \\
\text { thoracotomy }\end{array}$ \\
\hline 5 & Chester & 1959 & 46 & M & 22 & $\begin{array}{l}\text { Left-sided } \\
\text { chest pain }\end{array}$ & $10 \times 7.5 \times 3.0$ & $\begin{array}{l}\text { Exploratory } \\
\text { thoracotomy }\end{array}$ \\
\hline 6 & Perrin & 1960 & 56 & M & NR & $\begin{array}{l}\text { Right-sided } \\
\text { chest pain }\end{array}$ & $9 \times 7 \times 1.5$ & $\begin{array}{l}\text { Exploratory } \\
\text { thoracotomy }\end{array}$ \\
\hline 7 & Kyllonen & 1961 & 42 & $\mathrm{~F}$ & NR & $\begin{array}{l}\text { Left-sided } \\
\text { chest pain }\end{array}$ & $3 \times 2.5 \times 2.5$ & $\begin{array}{l}\text { Exploratory } \\
\text { thoracotomy }\end{array}$ \\
\hline 8 & Chipman & 1962 & 40 & $M$ & $N R$ & $\begin{array}{l}\text { Left-sided } \\
\text { chest pain }\end{array}$ & $7.5 \times 5 \times 4.5$ & $\begin{array}{l}\text { Exploratory } \\
\text { thoracotomy }\end{array}$ \\
\hline 9 & Kyllonen & 1964 & 51 & $M$ & NR & $\begin{array}{l}\text { Left-sided } \\
\text { chest pain }\end{array}$ & $2.5 \times 5$ & $\begin{array}{l}\text { Exploratory } \\
\text { thoracotomy }\end{array}$ \\
\hline 10 & Behrendt & 1968 & 23 & $\mathrm{~F}$ & $\begin{array}{c}\text { Moderately } \\
\text { obese }\end{array}$ & $\begin{array}{l}\text { Left-, then right-sided } \\
\text { chest pain }\end{array}$ & $4.5 \times 1.5 \times 1$ & $\begin{array}{l}\text { Exploratory } \\
\text { thoracotomy }\end{array}$ \\
\hline 11 & Takkunen & 1970 & 50 & $M$ & NR & $\begin{array}{l}\text { Left-sided } \\
\text { chest pain }\end{array}$ & 1.5 & $\begin{array}{l}\text { Exploratory } \\
\text { thoracotomy }\end{array}$ \\
\hline 12 & Wychulis & 1971 & 52 & $M$ & Obese & $\begin{array}{c}\text { Left-sided } \\
\text { chest pain (3 cases) }\end{array}$ & $5 \times 2.5 \times 2$ & $\begin{array}{l}\text { Exploratory } \\
\text { thoracotomy }\end{array}$ \\
\hline 13 & Wychulis & 1971 & 63 & $M$ & Obese & $\begin{array}{c}\text { Right-sided } \\
\text { chest pain (1 cases) }\end{array}$ & - & $\begin{array}{l}\text { Exploratory } \\
\text { thoracotomy }\end{array}$ \\
\hline 14 & Wychulis & 1971 & 64 & $M$ & Obese & $\begin{array}{l}\text { Left-sided } \\
\text { chest pain }\end{array}$ & $8 \times 4.5 \times 1.5$ & $\begin{array}{l}\text { Exploratory } \\
\text { thoracotomy }\end{array}$ \\
\hline 15 & Wychulis & 1971 & 47 & $\mathrm{~F}$ & Obese & $\begin{array}{l}\text { Left-sided } \\
\text { chest pain }\end{array}$ & - & $\begin{array}{l}\text { Exploratory } \\
\text { thoracotomy }\end{array}$ \\
\hline 16 & Webster & 1974 & 58 & $\mathrm{~F}$ & 28.2 & $\begin{array}{l}\text { Left-sided } \\
\text { chest pain }\end{array}$ & $3 \times 1.5$ & $\begin{array}{l}\text { Exploratory } \\
\text { thoracotomy }\end{array}$ \\
\hline 17 & Stephens & 1988 & 37 & $\mathrm{~F}$ & 19.7 & $\begin{array}{l}\text { Right-sided } \\
\text { chest pain }\end{array}$ & $4 \times 1.5 \times 1$ & $\begin{array}{l}\text { Exploratory } \\
\text { thoracotomy }\end{array}$ \\
\hline 18 & Bensard & 1990 & 67 & $\mathrm{~F}$ & Non-obese & $\begin{array}{l}\text { Left-sided } \\
\text { chest pain }\end{array}$ & $3 \times 3$ & $\begin{array}{l}\text { Exploratory } \\
\text { thoracotomy }\end{array}$ \\
\hline 19 & Inoue & 2000 & 55 & $M$ & 25.9 & $\begin{array}{l}\text { Left-sided } \\
\text { chest pain }\end{array}$ & $5 \times 3.5 \times 2$ & $\begin{array}{l}\text { Video-assisted } \\
\text { thoracic surgery } \\
\text { (VATS) }\end{array}$ \\
\hline 20 & Takao & 2004 & 45 & $M$ & NR & Hemoptysis & $N R$ & $\begin{array}{c}\text { Biopsy of } \\
\text { pericardial fat }\end{array}$ \\
\hline 21 & Pineda & 2005 & 54 & $\mathrm{~F}$ & Non-obese & Chest pain & $N R$ & $\begin{array}{c}\text { Analgesics, } \\
\text { documented } \\
\text { radiologic follow-up }\end{array}$ \\
\hline 22 & Pineda & 2005 & 50 & $M$ & Non-obese & $\begin{array}{l}\text { Left-sided } \\
\text { chest pain }\end{array}$ & $N R$ & $\begin{array}{l}\text { Exploratory } \\
\text { thoracotomy }\end{array}$ \\
\hline 23 & Lee & 2007 & 45 & $M$ & 22.3 & $\begin{array}{l}\text { Intermittent dizziness, } \\
\text { pitting edema }\end{array}$ & $6 \times 5$ & $\begin{array}{l}\text { Exploratory } \\
\text { thoracotomy }\end{array}$ \\
\hline $24 V$ & Van den Heuvel & 2010 & 55 & $\mathrm{~F}$ & $N R$ & Left-sided chest pain & $N R$ & Analgesics \\
\hline 25 & Present case & 2011 & 70 & $M$ & 34.8 & $\begin{array}{l}\text { Left-sided chest } \\
\text { and shoulder pain }\end{array}$ & $6,4 \times 2.5$ & $\begin{array}{c}\text { Analgesics, } \\
\text { documented } \\
\text { radiologic follow-up }\end{array}$ \\
\hline
\end{tabular}

*Year of publication 
required surgical resection for diagnosis. Pathologically, associated lesions resemble necrotic tissue more commonly found in epiploic appendages of the colon or in the breast [1], with a predominance of lipid-filled macrophages, inflammatory changes, and extravasation of blood [2]. Only 24 previous cases (see Table I) have been described in the medical literature [1-16]. However, of these 24 cases, 22 required surgical excision and histologic examination for diagnosis. Only one prior case [7] involved a tentative diagnosis of EPFN, symptomatic treatment, and documented resolution of the lesion without surgery.

The pathogenesis of EPFN remains unclear. Potential causes include torsion of a pedicle, a preexisting structural abnormality that may make tissue more vulnerable to necrosis, and heavy lifting, which could cause intravascular pressure changes significant enough to cause hemorrhage into adipose tissue. The associated pain of epipericardial fat necrosis is almost always pleuritic - sharp in quality, with intermittent peaks. In general, patients typically experience acute pleuritic chest pain. The pain has been described as leftsided in 16 patients [1-8, 11-13, 17], right-sided in $5[1,3,9,10,18]$, and was without further clear description in 3 cases $[7,15,16]$. In most instances, the onset is acute, on the order of days to weeks, however in uncommon cases, the pain can persist for close to one year [1, 3]. EKGs are normal, with rare exception $[2,6,9]$. Cardiac enzymes, leukocyte counts, and other laboratory tests, when performed, have been non-contributory.

Diaphoresis and syncope can complicate the presentation. Cases have generally been unrelated to trauma or infection. Obesity has been suggested as a risk factor, but from our literature review, only $42 \%$ (10 out of 24 patients) were described as being at least moderately obese. Still, the volume of pericardial fat is typically increased in overweight individuals [19]. Narrowing the differential diagnosis can be difficult, given the generally nonspecific presentation of EPFN, and radiographic similarities between fat necrosis and other fat-containing masses, including liposarcoma and lipoma. Historically, this ambiguity made surgical management essential [1, 3, 4, 9]. With advanced $C T$, important EPFN findings can include an encapsulated fatty lesion, with or without adjacent pericardial thickening [7]. Fat stranding in the lesion may assist in diagnosis. Chest pain in tandem with these radiographic features is highly suggestive of EPFN [7]. Although rare, it is important to consider this benign entity, based on its usual clinical presentation, combined with negative markers for $\mathrm{MI}$ and other cardiopulmonary pathology. A paracardiac density or opacity with or without a cardiophrenic or adjacent pleural effusion on routine chest film can suggest the diagnosis and prompt further evaluation with chest CT.

In conclusion, first described by Jackson, Clagett, and McDonald in 1957 [3] the definitive diagnosis of epipericardial fat necrosis has generally required surgical resection and pathologic examination. Of 24 previous cases, the case we presented represents only the second case of EPFN diagnosed and followed through resolution, using chest CT imaging studies alone. Given this prior report combined with the clinical course of our patient, we have presented additional evidence that strengthens the case for conservative management of this condition, and suggests that although EPFN is rare, it may be under-diagnosed. Both radiologists and practicing clinicians should be aware of the clinical presentation of this uncommon but benign condition and include it in their differential diagnosis of chest pain.

\section{References}

1. Wychulis AR, Connolly DC, McGoon DC. Pericardial cysts, tumors, and fat necrosis. J Thorac Cardiovasc Surg 1971; 62: 294.

2. Bensard DD, St. Cyr JA, Johnston MR. Acute pleuritic chest pain and lung mass in an elderly woman. Chest 1990; 97: 1473-4.

3. Jackson RC, Clagett OT, MCDonald JR. Pericardial fat necrosis: report of three cases. J Thorac Surg 1957; 33: 723-9.

4. Chester MH, Tully JB. Acute pericardial fat necrosis; report of a case. J Thorac Cardiovasc Surg 1959; 38: 62-6.

5. Kasserman WH. Pericardial fat necrosis: an unusual entity: a case report. J Thorac Surg 1958; 35: 689-91.

6. Inoue S, Fujino S, Tezuka N, et al. Encapsulated pericardial fat necrosis treated by video-assisted thoracic surgery: Report of a case. Surg Today 2000; 30: 739-43.

7. Pineda V, Caceres J, Andreu J, Vilar J, Domingo ML. Epipericardial fat necrosis: radiologic diagnosis and follow-up. Am J Roentgenol 2005; 185: 1234.

8. Takkunen J, Karkola P, Larmi T. Pericardial fat necrosis. report of a case diagnosed before thoracotomy. Ann Chir Gynaecol Fenn 1970; 59: 53-5.

9. Behrendt DM, Scannell JG. Pericardial fat necrosis. an unusual cause of severe chest pain and thoracic "tumor". N Engl J Med 1968; 279: 473-5.

10. Perrin MB. Pericardial fat necrosis. Can J Surg 1960; 4 : 76-8.

11. Kyllonen KE, Perasalo O. Acute pericardial fat necrosis. Acta Chir Scand 1961; 122: 275.

12. Chipman CD, Aikens RL, Nonamaker EP. Pericardial fat necrosis. Can Med Assoc J 1962; 86: 237.

13. Kylloenen KE. A case of pericardial fat necrosis simulating tumour of the lung. Acta Chir Scand 1964; 128: 778-80.

14. Webster MW Jr, Bahnson HT. Pericardial fat necrosis. case report and review. J Thorac Cardiovasc Surg 1974; 67: 430-3.

15. Takao H, Yamahira K, Watanabe T. Gallium uptake in pericardial fat necrosis. Clin Nucl Med 2004; 29: 378.

16. Lee BY, Song KS. Calcified chronic pericardial fat necrosis in localized lipomatosis of pericardium. Am J Roentgenol 2007; 188: W21. 
17. van den Heuvel DA, van Es HW, Cirkel GA, Bos WJ. Acute chest pain caused by pericardial fat necrosis. Thorax 2010; 65: 188.

18. Stephens DA, Kocab F. Pericardial fat necrosis. J Thorac Cardiovasc Surg 1988; 95: 727-9.

19. Pineda V, Andreu J, Cáceres J, Merino X, Varona D, Domínguez-Oronoz R. Lesions of the cardiophrenic space: Findings at cross-sectional Imaging1. Radiographics 2007; 27: 19 . 